\title{
Efeito do número de inseminações artificiais sobre a fertilidade de éguas inseminadas com sêmen asinino diluído e resfriado a $5^{\circ} \mathrm{C}$ por 12 horas de armazenamento
}

\author{
[Effect of the number of inseminations/cycle on fertility of inseminated mares with jackass \\ semen diluted and cooled at $5^{\circ} \mathrm{C}$ for 12 hours] \\ R. Rossi ${ }^{1}$, J.M. Silva Filho ${ }^{2}$, M.S. Palhares $^{2}$, E.A. Ribeiro ${ }^{3}$, Y.F. Resende ${ }^{4}$, F.R. Anjos ${ }^{5}$ \\ ${ }^{1}$ Aluno de pós-graduação - Escola de Veterinária - Universidade Federal de Minas Gerais - Belo Horizonte, MG \\ ${ }^{2}$ Escola de Veterinária - Universidade Federal de Minas Gerais - Belo Horizonte, MG \\ ${ }^{3}$ Médico veterinário autônomo \\ ${ }^{4}$ Aluna de graduação - Escola de Veterinária - PUC-Minas - Betim, MG \\ ${ }^{5}$ Aluna de graduação - Escola de Veterinária - Universidade Federal de Minas Gerais - Belo Horizonte, MG
}

\begin{abstract}
RESUMO
Estudou-se o efeito do número de inseminações sobre a fertilidade de éguas inseminadas com sêmen asinino diluído, resfriado e armazenado. Os ciclos foram acompanhados por palpação retal e rufiação, sendo as inseminações realizadas às terças, quintas e sábados (três vezes por semana), a partir da detecção de um folículo de 3,0 a $3,5 \mathrm{~cm}$ de diâmetro, em um dos ovários, até a ovulação. O sêmen de cinco jumentos da raça Pêga foi diluído nos diluidores de leite em pó desnatado-glicose ou glicina-gema de ovo, resfriado a $5^{\circ} \mathrm{C}$ e armazenado por 12 horas, sendo a dose inseminante de aproximadamente $400 \times 10^{6}$ espermatozoides móveis (no momento da diluição final, pré-resfriamento). De acordo com o número de inseminações artificiais (IA)/ciclo, os resultados de 195 ciclos, referentes a 140 éguas, foram agrupados em 1IA, 2IA e 3 ou mais IA. As taxas de concepção, ao primeiro ciclo, foram de 50,0\%, 62,1\% e de $41,0 \%$ e, após quatro ciclos, de $47,7 \%, 55,6 \%$ e de $42,9 \%$ para 1IA, 2IA e 3 ou mais IA, respectivamente $(\mathrm{P}>0,05)$. O número de IA/ciclo, utilizando sêmen asinino resfriado, não teve efeito sobre a fertilidade das éguas.
\end{abstract}

Palavras-chave: jumento, número de inseminações, resfriamento do sêmen, taxa de concepção

\begin{abstract}
The effect of the number of inseminations/cycles on the fertility of inseminated mares with diluted, cooled and stored jackass semen was studied. The cycles were controlled by rectal palpation and teasing, and mares were inseminated every Tuesday, Thursday and Saturday (three times/week), from the detection of a 3.0 to $3.5 \mathrm{~cm}$ follicle diameter in one of the ovaries until ovulation. The semen of five Pêga jackasses was diluted in skim milk-glucose or in egg yolk-glicine diluter and cooled at $5^{\circ} \mathrm{C}$ for 12 hours, with the inseminate dose of $400 \times 10^{6}$ motile spermatozoa (at the moment of the final dilution, before cooling). The results of 195 cycles of 140 mares were grouped, in accordance with the number of artificial inseminations (AI)/cycle in: IAI, 2AI and 3 or more AI. The conception rates for the first cycle were $50.0 \%, 62.1 \%$ and $41.0 \%$, and after four cycles they were $47.7 \%, 55.6 \%$ and $42.9 \%$, respectively for $1 \mathrm{AI}$, $2 A I$ and 3 or more AI $(p>0.05)$. The number of AI/cycles, using cooled jackass semen, had no influence on fertility.
\end{abstract}

Keywords: jackass, number of inseminations, cooling semen, conception rate

Recebido em 17 de maio de 2010

Aceito em 23 de novembro de 2011

E-mail: raissarossi@hotmail.com 


\section{INTRODUÇÃO}

A literatura é controversa no que diz respeito ao número ideal de inseminações a ser realizado por ciclo. Assim, já em 1937, havia preocupação em realizar sucessivas intervenções uterinas nas éguas, aumentando a possibilidade de infecção (Caslick, 1937). Acredita-se que, no manejo reprodutivo equino, o maior número de cobrições ou de inseminações possa influenciar negativamente a fertilidade das fêmeas, em razão do aumento da exposição do sistema genital predispondo-o à ocorrência de contaminação iatrogênica, principalmente em éguas susceptíveis à endometrite (Brinsko e Varner, 1993).

Buiten et al. (2003), ao envolverem éguas pônei, observaram que o menor número de inseminações - uma ou duas - apresentou melhores resultados do que três ou mais inseminações por ciclo - 77 ou $71 \%$ vs $64 \%$, respectivamente - considerando-se a taxa de não retorno aos 28 dias.

Contudo, Bristol (1982), ao utilizar 20 éguas sincronizadas e colocadas em uma área comum a um garanhão de seis anos de idade, observou que $85 \%$ (17/20) ficaram gestantes, embora fossem cobertas, em média, 4,6 vezes durante o primeiro ciclo. De forma similar, Voss et al. (1982) também demonstraram que o maior número de inseminações por ciclo não exerceu influência negativa sobre a fertilidade, pois não ocorreu infecção ou desenvolvimento de endometrite, mesmo quando duas éguas foram inseminadas por 27 ou 34 vezes, no mesmo ciclo, quando ambas se tornaram gestantes. Além disso, verificaram que as éguas gestantes foram inseminadas mais vezes por ciclo em relação às que não conceberam, 3,4 vs 2,8, respectivamente.

A eficiência de um programa reprodutivo pode ser conseguida com a redução do número de inseminações. Assim, apesar de alguns trabalhos demonstrarem melhor eficiência associada a maior número de cobrições ou inseminações (Voss et al., 1982; Squires et al., 1998; Sieme et al., 2003), a maioria dos estudos não demonstrou qualquer efeito dessa variável sobre a fertilidade das fêmeas equinas (Pickett e Shiner, 1994; Valle et al., 2000; Brandão et al., 2003; Xavier et al., 2010).
Outro ponto relevante a ser destacado é a escassez de trabalhos com sêmen de asininos, também controversos, que avaliaram a influência do número de inseminações sobre a fertilidade de equídeos, incluindo o cruzamento industrial de éguas com jumentos (Palhares et al., 1986; Silva, 1988; Ferreira, 1993; Leite, 1994).

A fertilidade das éguas parece mais dependente do adequado intervalo IA-ovulação do que do número de inseminações artificiais realizadas, mesmo com concentração espermática adequada para cada tipo de sêmen utilizado - in natura, resfriado, resfriado e transportado ou congelado (Silva Filho, 1994; Sieme et al., 2003).

O objetivo deste trabalho foi verificar o efeito do número de inseminações artificiais, por ciclo, sobre a fertilidade de éguas inseminadas com sêmen asinino resfriado.

\section{MATERIAL E MÉTODOS}

O trabalho foi desenvolvido entre os meses de outubro de 2007 e janeiro de 2008, utilizando-se 195 ciclos estrais referentes a 140 éguas mestiças, com 2,5 a 19 anos de idade, distribuídas de forma inteiramente ao acaso, por meio de sorteio, após agrupamento por idade e por categoria reprodutiva em: potra, égua solteira, égua parida ou no "cio do potro".

As éguas foram examinadas por meio de palpação transretal, diariamente, a partir da detecção de um folículo de 2,0 a $2,5 \mathrm{~cm}$ de diâmetro, em um dos ovários. A seguir, foram rufiadas quando necessário. As inseminações foram realizadas quando da detecção de um folículo com diâmetro de 3,0 a $3,5 \mathrm{~cm}$, em um dos ovários, até a ovulação, sendo o sêmen depositado no corpo do útero por via intravaginal profunda. Para o diagnóstico de gestação, palpação transretal e rufiação de retorno foram realizadas a partir do $15^{\circ}$ dia pós-ovulação, complementadas pelo uso de ultrassonografia, realizada a cada 14 dias.

Foram utilizados cinco jumentos da raça Pêga como doadores de sêmen, previamente avaliados. O sêmen foi coletado por meio de vagina artificial modelo Hannover, às terças, quintas e sábados (três vezes/semana). Após avaliação da motilidade e do vigor espermáticos, realizou-se a contagem das células na câmara de Newbauer, 
para cálculo da concentração, com posterior diluição do sêmen em diluidor à base de leite em pó desnatado-glicose (Kenney et al., 1983) ou de glicina-gema de ovo (Foote, 2002). As doses inseminantes, de $20 \mathrm{~mL}$ e $400 \times 10^{6}$ espermatozoides móveis, foram resfriadas a $5^{\circ} \mathrm{C}$ por 12 horas de armazenamento, em contêiner especial, apresentando queda de temperatura lenta, entre $18-8^{\circ} \mathrm{C}$, de $0,07^{\circ} \mathrm{C} /$ minuto (Palhares, 1997).

Para análise do efeito do número de inseminações sobre a fertilidade, agruparam-se os dados segundo o número de inseminações realizadas por ciclo em: 1IA, 2IA e 3 ou mais IA. $\mathrm{O}$ número de inseminações em cada ciclo foi consequência da duração do cio da égua, o que resultou em número de repetições diferente para cada grupo.

A eficiência de prenhez foi calculada pela conversão dos dados qualitativos em quantitativos, como proposto por Voss et al. (1975). Os dados foram submetidos à análise de variância, e a comparação de médias foi feita pelo teste SNK. Os dados quantitativos foram também submetidos à análise de variância e teste SNK. Os dados proporcionais - número de ciclos/concepção, taxa de concepção/ciclo, taxa de concepção total e ciclos/égua gestante foram analisados por dispersão de frequência e comparados pelo teste do qui-quadrado para detecção de diferenças entre os grupos.

\section{RESULTADOS E DISCUSSÃO}

As taxas de concepção ao primeiro ciclo - 64,5\% vs $61,1 \%$ - e as taxas de concepção/ciclo $63,6 \%$ vs $54,6 \%$ - foram similares $(\mathrm{P}>0,05)$ para os diluidores de leite em pó desnatado-glicose ou de glicina-gema de ovo, respectivamente. Também não se observou efeito da categoria reprodutiva e do intervalo IA-ovulação sobre a eficiência reprodutiva das éguas (Rossi, 2008). Da mesma forma, para as fêmeas inseminadas uma, duas e três ou mais vezes por ciclo, não foram observadas diferenças $(\mathrm{P}>0,05)$ para as taxas de concepção, ao primeiro ciclo, e as de concepção/ciclo (Tab. 1), bem como para o número de ciclos/concepção e eficiência de prenhez (Tab. 2).

Tabela 1. Número de ciclos e taxa de concepção de éguas inseminadas com sêmen de jumento, de acordo com o número de inseminações, por ciclo estral

\begin{tabular}{|c|c|c|c|c|c|c|c|c|}
\hline \multirow[b]{2}{*}{ Ciclo } & \multicolumn{2}{|r|}{$1 \mathrm{IA}$} & \multicolumn{2}{|r|}{2 IA } & \multicolumn{2}{|r|}{$\geq 3 \mathrm{IA}$} & \multicolumn{2}{|r|}{ Total } \\
\hline & $\begin{array}{l}\text { № de } \\
\text { ciclos }\end{array}$ & $\begin{array}{c}\text { Taxa } \\
\text { concepção(\%) }\end{array}$ & $\begin{array}{l}\text { № de } \\
\text { ciclos }\end{array}$ & $\begin{array}{c}\text { Taxa } \\
\text { concepção(\%) }\end{array}$ & $\begin{array}{l}\text { № de } \\
\text { ciclos }\end{array}$ & $\begin{array}{c}\text { Taxa } \\
\text { concepção(\%) }\end{array}$ & $\begin{array}{l}\text { № de } \\
\text { ciclos }\end{array}$ & $\begin{array}{c}\text { Taxa } \\
\text { concepção(\%) }\end{array}$ \\
\hline 1 & $44(22)$ & 50,00 & $58(36)$ & 62,07 & $39(16)$ & 41,03 & $141(74)$ & 52,40 \\
\hline 2 & $13(7)$ & 53,85 & $18(7)$ & 38,89 & $9(4)$ & 44,44 & $40(18)$ & 45,00 \\
\hline 3 & $7(2)$ & 28,57 & $4(1)$ & 25,00 & $1(1)$ & 100,00 & $12(4)$ & 33,33 \\
\hline 4 & $1(0)$ & 0,00 & $1(1)$ & 100,00 & $0(0)$ & 0,00 & $2(1)$ & 50,00 \\
\hline Total & $65(31)$ & 47,69 & $81(45)$ & 55,56 & $49(21)$ & 42,86 & $195(97)$ & 49,70 \\
\hline
\end{tabular}

Valores entre parênteses referem-se aos ciclos gestantes.

1IA: uma inseminação/ciclo; 2IA: duas inseminações/ciclo; $\geq 3$ IA: três ou mais inseminações/ciclo.

As variáveis de controle, tais como idade da égua, volume de sêmen, volume de diluidor, concentração espermática/dose, tempo de colheita-diluição, tempo de colheita-abertura e tempo de colheita-IA foram semelhantes $(\mathrm{P}>0,05)$ entre os grupos (Tab. 2). No entanto, o tempo de colheita-resfriamento diferiu $(\mathrm{P}<0,05)$ entre os grupos, embora a diferença seja irrelevante do ponto de vista prático. Vale salientar que o tempo da colheita à diluição do sêmen foi de, aproximadamente, 20 minutos, de maior duração que o recomendado por Palmer (1984), ao observar que o tempo curto, de três minutos, da coleta à diluição, foi um fator de importância na manutenção da qualidade espermática, visto que o aumento do tempo para 10 minutos reduziu significativamente a motilidade espermática.

Como esperado, houve diferença $(\mathrm{P}<0,05)$ entre os grupos com relação ao número de IA/ciclo, ao número de IA/ciclo positivo e ao número de IA/ciclo negativo (Tab. 2). Ferreira (1993), ao utilizar sêmen asinino fresco diluído ou resfriado por 24 ou 48 horas, respectivamente, obteve número de IA/ciclo positivo de 1,58; 1,82 e de 
1,79, em éguas. Já Palhares et al. (1986), ao utilizarem sêmen da mesma espécie, obtiveram 1,6 IA/jumenta gestante e 2,8 IA/jumenta vazia, quando avaliaram a relação entre o número de IA e a fertilidade.

Silva (1988), ao trabalhar com 240 éguas, utilizou sêmen fresco diluído de jumentos da raça Pêga, em três estações consecutivas. O número de IA/égua foi de 2,68, 3,26 e de 2,19, e o número de IA/égua prenha de 3,93, 3,96 e de 2,38, respectivamente, para as estações 1984/85, 1985/86 e 1986/87. A redução das inseminações, no transcorrer do trabalho, ocorreu em razão da maior utilização de inseminações após a ovulação, demonstrando melhor eficiência do manejo reprodutivo, quando empregado corretamente.

Tabela 2. Variáveis de controle e de resultados de éguas inseminadas com sêmen diluído e resfriado, agrupadas de acordo com o número de inseminações artificiais

\begin{tabular}{|c|c|c|c|}
\hline \multirow{2}{*}{ Variável } & \multicolumn{3}{|c|}{ Número de Inseminações } \\
\hline & $1(n=47)$ & $2(n=61)$ & $\geq 3(n=32)$ \\
\hline № de ciclos & 65 & 81 & 49 \\
\hline Idade das éguas (anos) & $9,52 \pm 4,03$ & $9,77 \pm 3,69$ & $10,09 \pm 4,26$ \\
\hline № de ciclos/égua & $1,49 \pm 0,78$ & $1,33 \pm 0,60$ & $1,25 \pm 0,51$ \\
\hline № IA/ciclo & $1,00 \pm 0,00 \mathrm{c}$ & $2,00 \pm 0,00 b$ & $3,29 \pm 0,71 \mathrm{a}$ \\
\hline Volume de sêmen (mL) & $2,72 \pm 1,32$ & $2,89 \pm 1,65$ & $3,03 \pm 1,70$ \\
\hline Volume de diluidor (mL) & $17,30 \pm 1,33$ & $17,13 \pm 1,67$ & $16,97 \pm 1,70$ \\
\hline Concentração Sptz /dose IA & $403,14 \pm 14,17$ & $401,36 \pm 7,48$ & $402,04 \pm 12,72$ \\
\hline Tempo colheita/diluição (min) & $19,17 \pm 3,40$ & $18,54 \pm 2,74$ & $19,32 \pm 3,39$ \\
\hline Tempo colheita/resfriamento (min) & $24,86 \pm 4,92 \mathrm{ab}$ & $23,93 \pm 2,84 b$ & $24,93 \pm 3,92 \mathrm{a}$ \\
\hline Tempo colheita/abertura (h) & $13,15 \pm 0,60$ & $13,20 \pm 0,79$ & $13,14 \pm 0,84$ \\
\hline Tempo colheita/IA (h) & $13,31 \pm 0,60$ & $13,34 \pm 0,79$ & $13,30 \pm 0,86$ \\
\hline № de IA/ciclo positivo & $1,00 \pm 0,00 \mathrm{c}$ & $2,00 \pm 0,00 \mathrm{~b}$ & $3,33 \pm 0,80 \mathrm{a}$ \\
\hline № de IA/ciclo negativo & $1,00 \pm 0,00 \mathrm{c}$ & $2,00 \pm 0,00 \mathrm{~b}$ & $3,25 \pm 0,65 a$ \\
\hline № de ciclos/concepção & 2,10 & 1,80 & 2,33 \\
\hline Taxa de concepção/ciclo & 0,48 & 0,56 & 0,43 \\
\hline № de ciclos/égua gestante & $1,35 \pm 0,61$ & $1,27 \pm 0,62$ & $1,29 \pm 0,56$ \\
\hline Eficiência de prenhez & $4,43 \pm 4,75$ & $5,26 \pm 4,82$ & $4,04 \pm 4,77$ \\
\hline
\end{tabular}

Médias seguidas por letras diferentes, na mesma linha, diferem entre si $(\mathrm{P}<0,05)$.

Sptz - espermatozoides $\times 10^{6}$.

A redução do número de inseminações, no presente trabalho, não causou prejuízos à fertilidade, já que a taxa de concepção, ao primeiro ciclo - de $50,0 \% ; 62,1 \%$ e $41,0 \%$ - e a de concepção/ciclo - de 47,7\%; $55,6 \%$ e $42,9 \%$ - não diferiram $(\mathrm{P}>0,05)$ para éguas inseminadas uma, duas ou três ou mais vezes/ciclo, de forma similar aos trabalhos realizados por Palhares (1997), Valle et al. (2000) e Brandão et al. (2003). Esses autores utilizaram o mesmo manejo reprodutivo, com sêmen de garanhões, e não observaram qualquer relação $(\mathrm{P}>0,05)$ entre a taxa de concepção e o número de inseminações realizadas por ciclo. Da mesma forma, mas utilizando sêmen fresco diluído de jumento, Leite (1994) observou que o momento bem como a frequência de inseminações não influenciaram a taxa de gestação.

Ferreira (1993) relatou que o aumento do número de inseminações, de uma para duas ou mais vezes, bem como a redução do intervalo de 24-48 para 0-24 horas influenciaram significativamente a taxa de concepção, quando inseminaram éguas com sêmen asinino resfriado a $5^{\circ} \mathrm{C}$, por 24 a 48 horas. $\mathrm{O}$ efeito do momento de inseminação - até 24 horas pré-ovulação vs 
24-48 horas pré-ovulação - ficou mais evidente $(\mathrm{P}<0,05)$ quando associado a apenas uma IA. Assim, $85 \%$ das éguas conceberam quando submetidas a uma IA, realizada dentro de 24 horas da ovulação, ao passo que apenas $53,6 \%$ ficaram gestantes, quando inseminadas no intervalo 24-48 horas de sua ocorrência. Quando as éguas foram submetidas a duas inseminações por ciclo, não se observou efeito do intervalo IAovulação sobre a fertilidade, que foi de $93,8 \%$ para as inseminadas dentro de 24 horas da ovulação e de $100,0 \%$ para as inseminadas no intervalo 24-48 horas de sua detecção.

Alguns fatores devem ser ajustados para se determinar o número de inseminações a serem realizadas, já que há associação entre frequência de inseminações, concentração espermática (Pickett et al., 1987) e intervalo IA-ovulação (Woods et al., 1990). Desse modo, com base na literatura e nos resultados deste experimento, há que se enfatizar ser a fertilidade das fêmeas mais dependente do adequado intervalo IA-ovulação do que do número de inseminações artificiais realizadas por ciclo. No entanto, para se obter redução do número de inseminações, sem que ocorram prejuízos à fertilidade, é necessário utilizar sêmen de reprodutores que apresentem alta viabilidade espermática, até o momento da fecundação, e desenvolver programas mais precisos de controle folicular, que permitam a realização da inseminação o mais próximo possível da ovulação. Assim, o incremento da eficiência de um programa reprodutivo pode ser conseguido limitando-se esse número, com consequente uso mais racional do reprodutor, menor gasto com doses de sêmen e material utilizado, redução dos riscos de endometrite (Brinsko e Varner, 1993), além de reduzir o deslocamento de fêmeas até o curral, o que proporcionaria melhor manejo reprodutivo na propriedade.

\section{CONCLUSÕES}

Como o número de inseminações artificiais não influenciou a fertilidade de éguas inseminadas com sêmen asinino resfriado, quando possível deve-se, por meio do melhor controle da ovulação da égua, utilizar apenas uma inseminação, com intuito de aumentar a eficiência do programa reprodutivo empregado.

\section{AGRADECIMENTOS}

À FAPEMIG, pelo financiamento parcial deste trabalho; ao Sr. Renato Resende pela cessão dos animais e instalações da Fazenda do Vau; à Hertape Calier, na pessoa da Dra. Marcela Roscoe, pelos medicamentos concedidos para o trabalho.

\section{REFERÊNCIAS}

BRANDÃO, F.Z; $\quad$ SILVA FILHO, J.M.; PALHARES, M.S. et al. Efeito da concentração espermática e do número de inseminações artificiais sobre a fertilidade de éguas inseminadas com sêmen fresco diluído. Arq. Bras. Med. Vet. e Zootec., v.55, p.61-67, 2003. Disponível em: <http://www.scielo.br>. Acessado em: 4 de abr. de 2008.

BRINSKO, S.P.; VARNER, D.D. Artificial insemination. In: McKINNON, A.O.; VOSS, J.L. Equine Reproduction. Malvern: Lea \& Febiger, 1993. p.790-796.

BRISTOL, F. Breeding behaviour of stallion at pasture with 20 mares in synchronized oestrus. $J$. Reprod. Fertil., v.32, suppl., p.71-77, 1982.

BUITEN, A.V.; WESTERS, P.; COLENBRANDER, B. Male, female and management risk factors for non-return to service in dutch mares. Prev. Vet. Med., v.61, p.17-26, 2003.

CASLICK, E.A. The vulva and vulvo-vagina orifice and its relation to the genital health of the thoroughbred mare. Cornell Vet., v.27, p.178187, 1937.

FERREIRA, M.F.L. Efeito de diluente e taxa de resfriamento sobre a motilidade espermática $e$ fertilidade do sêmen de jumento (equus asinus). 1993. 94f. Dissertação (Mestrado em Medicina Veterinária) - Escola de Veterinária, Universidade Federal de Minas Gerais, Belo Horizonte, MG.

FOOTE, R.H. Whithin-Herd use of boar semen at $5^{\circ} \mathrm{C}$, with a note eletronic monitoring of oestrus. Reprod. Dom. Anim., v.37, p.61-63, 2002.

KENNEY, R.M.; HURTGEN, J.P.; PIERSON, R. et al. Society for theriogenology manual for clinical fertility evaluation of the stallion. Hastings: Society for Theriogenology, 1983. 100 p. 
LEITE, S.V.F. Influência do número de espermatozoides de jumento (Equus asinus) sobre a taxa de prenhez em éguas (Equus cabalus) inseminadas a fresco. 1994. 52f. Dissertação (Mestrado em Medicina Veterinária) - Escola de Veterinária, Universidade Federal de Minas Gerais, Belo Horizonte, MG.

PALHARES, M.S. Influência de um novo container para o transporte do sêmen equino diluído e resfriado: I- Características termodinâmicas e funcionais, II- Desempenho reprodutivo de éguas inseminadas. 1997. $245 \mathrm{f}$. Dissertação (Doutorado em Ciência Animal) Escola de Veterinária, Universidade Federal de Minas Gerais, Belo Horizonte, MG.

PALHARES, M.S.; SILVA FILHO, J.M.; OLIVEIRA Jr., E.F. et al. Inseminação artificial em jumentas da raça Pêga com cio induzido pelo Dinoprost. Rev. Bras. Reprod. Anim., v.10, p.157-163, 1986.

PALMER, E. Factors affecting stallion semen survival and fertility. In: INTERNATIONAL CONGRESS ON ANIMAL REPRODUCTION AND ARTIFICIAL INSEMINATION, 10, 1984, Urbana. Proceedings... Urbana: University of Illinois, 1984. v.3, p.377-379.

PICKET, B.W.; SHINER, K.A. Recent developments in artificial insemination in horses. Liv. Prod. Sci., v.40, p.31-36, 1994.

PICKETT, B.W.; SQUIRES, E.L.; McKINNON, A.O. et al. Procedures for collection, evaluation and utilization of stallion semen for artificial insemination. Fort Collins: Colorado State University, 1987. 125p. Bulletin, 03.

ROSSI, R. Comparação de dois diluidores na fertilidade de éguas inseminadas com sêmen asinino a fresco ou resfriado. 2008. 209f. Dissertação (Mestrado em Medicina Veterinária) - Escola de Veterinária, Universidade Federal de Minas Gerais, Belo Horizonte, MG.

SIEME, H.; SCHAFER, T.; STOUT, T.A.E. et al. The effects of different insemination regimes on fertility in mares. Theriogenology, v.60, p.1153-1164, 2003.
SILVA, M.I.F. Viabilidade do uso da inseminação artificial em equinos em nível industrial. In: CONGRESSO BRASILEIRO DE REPRODUÇÃO ANIMAL. 7., Belo Horizonte, 1988. Anais... Belo Horizonte: Colégio Brasileiro de Reprodução Animal, 1988, p.47-63.

SILVA FILHO, J.M. Aspectos do manejo reprodutivo e do sêmen na inseminação artificial de éguas. 1994. 497f. Dissertação (Doutorado em Zootecnia) - Universidade Federal de Viçosa, Viçosa, MG.

SQUIRES, E.L.; BRUBAKER, J.K.; McCUE, P.M.

et al. Effect of sperm number and frequency of insemination on fertility of mares inseminated with cooled semen. Theriogenology, v.49, p.743749, 1998.

VALLE, G.R.; SILVA FILHO, J.M.; PALHARES, M.S. et al. Efeito do número de inseminações artificiais sobre a fertilidade de éguas inseminadas com sêmen diluído, resfriado a $14^{\circ} \mathrm{C}$ e transportado. Rev. Bras. Zootec., v.29, p.1721-1726, 2000.

VOSS, J.L.; PICKETT, B.W.; BACK, D.G. et al. Effect of rectal palpation on pregnancy rate of nonlactanting, normaly cycling mares. J. Anim. Sci., v.41, p.829-834, 1975.

VOSS, J.L.; SQUIRES, E.L.; PICKETT, B.W. et al. Effect of number and frequency of inseminations on fertility of mares. J. Reprod. Fertil., v.32, suppl., p.53-57, 1982.

WOODS, J.; BERGFELT, D.R.; GINTHER, O.J. Effects of time of insemination relative to ovulation on pregnancy rate and embryonic-loss rate in mares. Equine Vet. J., v.22, p.410-415, 1990.

XAVIER, I.L.G.S.; SILVA FILHO, J.M.; CARVALHO, G.R. et al. Efeitos do local de deposição do sêmen e do intervalo inseminação/ovulação sobre a fertilidade de éguas inseminadas com sêmen fresco diluído. Rev. Bras. Zootec., v.39, p.512-519, 2010. 